\title{
Evidence of molecular alignment fluctuations in aqueous dilute liquid crystalline media
}

\author{
Martti Louhivuori · Renee Otten · Tapio Salminen • \\ Arto Annila
}

Received: 6 January 2007 / Accepted: 19 July 2007/Published online: 15 August 2007

(C) Springer Science+Business Media B.V. 2007

\begin{abstract}
Protein dynamics can be studied by NMR measurements of aqueous dilute liquid crystalline samples. However, the measured residual dipolar couplings are sensitive not only to internal fluctuations but to all changes in internuclear vectors relative to the laboratory frame. We show that side-chain fluctuations and bond librations in the ps-ns time scale perturb the molecular shape and charge distribution of a small globular protein sufficiently to cause a noticeable variation in the molecular alignment. The alignment variation disperses the bond vectors of a conformational ensemble even further from the dispersion already caused by internal fluctuations of a protein. Consequently RDC-probed order parameters are lower than those obtained by laboratory frame relaxation measurements.
\end{abstract}

M. Louhivuori $(\bowtie) \cdot$ T. Salminen · A. Annila

Department of Physical Sciences, University of Helsinki, Gustaf Hällströmin katu 2, Helsinki 00014, Finland

e-mail: m.j.louhivuori@rug.nl

Present Address:

M. Louhivuori

Zernike Institute for Advanced Materials, University of

Groningen, Nijenborgh 4, Groningen 9747 AG, The Netherlands

R. Otten

Groningen Biomolecular Sciences and Biotechnology Institute, University of Groningen, Nijenborgh 4, Groningen 9747 AG,

The Netherlands

\section{A. Annila}

Institute of Biotechnology, University of Helsinki, Viikinkaari 1, Helsinki 00014, Finland

A. Annila

Department of Biosciences, University of Helsinki, Viikinkaari 1, Helsinki 00014, Finland
Keywords Liquid crystal NMR spectroscopy . Protein dynamics $\cdot$ Residual dipolar couplings

\section{Introduction}

Native proteins are customarily pictured as highly structured entities; a view that is supported by many lines of evidence. Nonetheless, all proteins exhibit internal motions on various time scales and amplitudes. Fast internal motions, i.e. bond vibrations and librations as well as peptide plane movements, take place in picoseconds to nanoseconds (Bouvignies et al. 2005; Kay et al. 1989; Lakomek et al. 2006). Slow internal motions, i.e. secondary structure and protein domain motions, occur at a sedate pace in microseconds to milliseconds. NMR coherence decays have revealed that bond librations and side-chain reorientations amount only to a small reduction in structural order. The associated Lipari-Szabo order parameter $\left(S_{L S}^{2}\right)$ (Lipari and Szabo 1982a, b) in well-structured parts of a protein is typically above 0.85 for amide moieties (Kay et al. 1989), 0.9 for backbone bonds (Ferrage et al. 2006) and $0.2-0.9$ for side-chain methyl groups depending on the length of the side-chain (Millet et al. 2002; Skrynnikov et al. 2002). On slower time scales, in the $\mu$ s-regime and above, relaxation dispersion and exchange rate measurements (Palmer 2004) have revealed larger but infrequent excursions away from the most populated states. Recently, residual dipolar coupling (RDC) data has revealed (Lakomek et al. 2005; Meiler et al. 2001; Tolman et al. 2001) in the intervening time-scale, below $\mu$ s and above $\tau_{c}$, anisotropic and correlated motions, that have been associated with order parameters in the range 0.6-1.0.

RDCs (Tjandra and Bax 1997) can be regarded as ideal motional probes as they respond explicitly to all motions 
of internuclear vectors. Non-zero RDCs arise first when molecular tumbling in solution is slightly anisotropic. To that end a dilute liquid crystalline (LC) medium is used to impose a subtle alignment on a biological macromolecule solute via steric and electrostatic interactions (Prestegard et al. 2004). RDC observables are, of course, averages over a dynamic conformational ensemble (Bax et al. 2001; Louhivuori et al. 2006). The high structural similarity among the most populated states prompts to think that on average all conformations would, to an excellent approximation, adopt a similar alignment. This is the implicit assumption underlying the many structural and dynamic data analyses of weak alignment NMR spectroscopy - a method that is widely used today in structural biology (Bax and Grishaev 2005; Blackledge 2005). Indeed it seems reasonable to imagine that the dispersion of molecular alignments among the conformations is small, perhaps negligible, in particular as no apparent inconsistencies have been noticed in protein structure determination. Furthermore it may also seem reasonable to use in the analysis of dynamics an effective alignment frame because usually the conformations are in a fast exchange compared to molecular reorientation.

In this study we inspect critically the concept of a common alignment frame for a protein structure on the basis of experimental data. We find that a variation in molecular alignment due to the ps-ns fluctuations can contribute significantly to the RDC-probed order parameter in the case of a small globular protein.

\section{Theory}

Protein motions captured by RDCs

A residual dipolar coupling reports essentially on the average direction of an internuclear vector, e.g. a chemical bond, relative to the main magnetic field of a NMR spectrometer. Both fast and slow internal motions perturb the directions of a chemical bond and since RDCs are measured as an average over a longer time period this reflects directly into the observed RDC. Besides internal motions, the molecule experiences also rotational and translational diffusion in the liquid sample. Anisotropic orientational sampling in a dilute liquid crystalline medium is the basis for observing a residual of a dipolar coupling (Tjandra and Bax 1997). In practice, it may not be possible to deconvolute the observed data to distinct motional modes, but their identification will clarify the analysis.

From a molecular perspective, i.e. from the perspective of an observer on a single molecule, external motions are incidental and of no particular interest - at least if we assume that molecule-medium interactions are negligible.
It would therefore be sufficient to consider any disorder present in molecular structures only in terms of internal motions (Fig. 1). The averaging due to the fast ps-ns bond fluctuations when inspected in the local sub-molecular frame is given by the aforementioned $S_{L S}^{2}$ values. The recently proposed (Bernadó and Blackledge 2004; Lakomek et al. 2006; Meiler et al. 2003; Peti et al. 2002; Tolman 2002) substantial and frequent fluctuation of secondary structures, i.e. $\alpha$-helices or $\beta$-sheets, relative to a molecular frame will cause additional down-scaling of the order parameter. We denote the corresponding order parameter as $S_{\alpha \beta}^{2}$ and note that it has been reported to range from 0.6 to 1.0 .

From an ensemble perspective, i.e. from the perspective of an observer regarding every molecule in the sample, both internal and external motions are manifestations of the state space of the system (Louhivuori et al. 2006). Internal motions cause the conformational diversity and external motions are responsible for the various orientations and positions the molecules inhabit. A conformational change is, from the ensemble perspective, tied to a change in the effective alignment of that molecule. This connection between the alignment and the conformation seems like non-sense from the molecular perspective since a conformational change does not instantly change the orientation of the molecule. Since RDCs are a time and ensemble average the ensemble

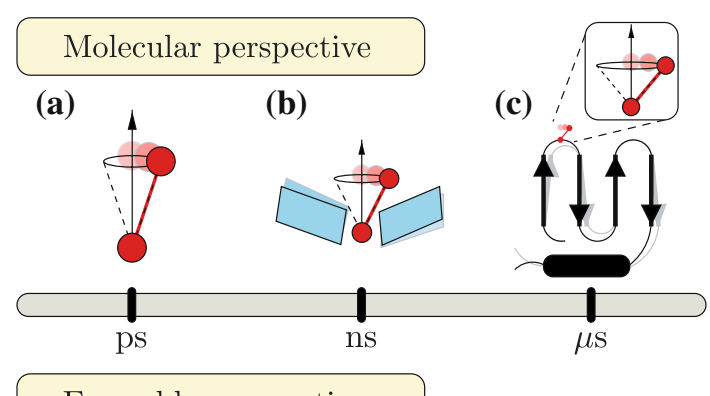

Ensemble perspective

(d)

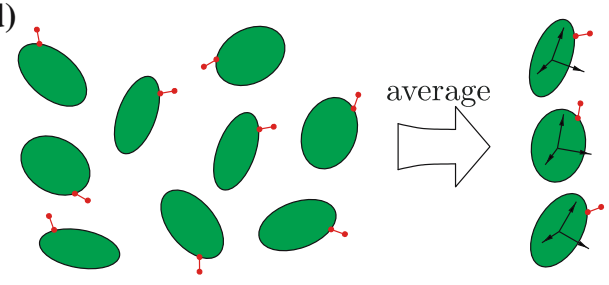

Fig. 1 In the molecular perspective internal motions like (a) bond fluctuations, (b) peptide plane motions and (c) secondary structure movements all contribute to disperse a chemical bond away from its average direction. (d) In the ensemble perspective both internal and external motions contribute to fill the available state space. In a RDC measurement the available state space is reduced to effective alignment tensors of the constituent conformations 
perspective is an appropriate choice. In the vicinity of a liquid crystalline particle conformations are not energetically equivalent (Louhivuori et al. 2006) and therefore e.g. some side-chain conformations are not in a fast exchange. This means that conformational changes due to internal motions couple to the alignment and appear as fluctuations in the effective alignment frame of a molecule. We mark the corresponding order parameter as $S_{\Omega}^{2}$. It should be emphasised that the alignment is not a property of a free molecule, i.e. a molecule in void, but rather a property of the whole sample including the LCparticles. $S_{\Omega}^{2}$ does not therefore correspond to any intrinsic molecular motion, even though it might appear as one if the molecular perspective is chosen for RDC data interpretation.

Finally, we remind that molecular tumbling in solution is the most effective averaging process with respect to the laboratory frame. The associated order parameter $S_{\tau}^{2}$ is on the order of $10^{-6}$ in a dilute LC-medium (Bax et al. 2001; Tjandra and Bax 1997). The order parameters are usually normalized relative to $S_{\tau}^{2}$.

\section{Dynamics modulated alignment}

We reason that the variation in molecular alignment originates from the dynamics-induced modulation of van der Waals and Coulomb interactions between the protein conformations and the nematogens (Louhivuori et al. 2006). It may appear paradoxical that a transient conformation could align as the internal ps-ns fluctuations are much faster than the molecular translational and rotational diffusion. This quandary is resolved as explained above in what is formally referred to as Boltzmann's ergodic hypothesis of the equivalence between time and ensemble averages. The suppression of some of the molecules internal fluctuations by the presence of the LC-particle imposes an unique alignment on each transient conformation. In other words, a transient conformation does not align per se but rather may only exist if it is energetically allowed. It is thus mistaken to justify the use of a single, effective alignment frame solely on the fact that internal fluctuations are much faster than translational or rotational diffusion.

It is clear then that dynamics modulated alignment (DMA) is a conceivable phenomenon and could very well be expressed in experimental data. We will therefore proceed to search for experimental evidence of it. Our analysis will be restricted to the ps-ns protein dynamics that is well characterized by NMR relaxation measurements. On such short time scales primarily bonds fluctuate and side-chains reorientate while motions of secondary structure elements are negligible (Palmer 2004).
Signature of dynamics modulated alignment

Of the many motions captured by residual dipolar couplings, bond fluctuations and the overall molecular tumbling are well understood. These two contributions should be removed from the total order to expose any additional motion attributable to either DMA or additional intrinsic motion, e.g. in the form of secondary structure fluctuations, or to both. If the additional motion is an exclusive property of the protein, i.e. independent of the LC-medium that was used in measurements, then it is likely evidence of the reported sub- $\mu$ s intrinsic secondary structure fluctuations. On the other hand, if a change in LCmedia makes a difference then the excess motion may just as well result from the alignment frame dispersion in DMA. Obviously neither secondary structure nor alignment fluctuations are mutually exclusive and indeed it is quite conceivable that both contribute to the observed additional motions. The presence of DMA does not therefore disprove for example the recent findings (Lakomek et al. 2005) of correlated backbone motions.

All order examined by RDCs is captured by an overall molecular measure called the general degree of order (GDO) (Tolman et al. 2001). We employ its squared value, $\vartheta^{2}=2 / 3 \sum \vartheta_{i j}^{2}$. The components $\vartheta_{i j}$ are obtained from the measured RDCs, e.g. by singular value decomposition (SVD) (Losonczi et al. 1999), using a set of equations

$R D C=\left(\frac{\mu_{o}}{4 \pi}\right) \frac{\gamma_{A} \gamma_{B} h}{2 \pi^{2} r_{A B}^{3}} \sum_{i j=\{x, y, z\}} \vartheta_{i j} c_{i} c_{j}$

where the refined structural model is given by $c_{i} c_{j}$, i.e. internuclear vectors in the Cartesian $\{x, y, z\}$ frame. The constants for the two nuclei $\mathrm{A}$ and $\mathrm{B}$ are the gyromagnetic ratios $\gamma_{\mathrm{A}}$ and $\gamma_{\mathrm{B}}$, the internuclear distance $r_{\mathrm{AB}}$ and as usual Planck's constant $h$ and the permeability of vacuum $\mu_{\mathrm{o}}$.

We aim to determine the ps-ns fluctuations, i.e. the $S_{L S}^{2}$ contribution to $\vartheta^{2}$, on the basis of the intrinsic difference between amide $(\mathrm{NH})$ and backbone $\left(\mathrm{C}^{\alpha} \mathrm{CO}\right)$ bond fluctuations. This difference is not large, but it is significant (Ferrage et al. 2006). We find from molecular dynamics (MD) simulations of ubiquitin and the B3 domain of protein $\mathrm{G}$ that the $\mathrm{NH}$ order parameter squared is on the average $\left\langle\vartheta_{\mathrm{NH}}^{2}\right\rangle_{Q}=0.878 \pm 0.002$ and the corresponding $\mathrm{C}^{\alpha} \mathrm{CO}$ value $\left\langle\vartheta_{\mathrm{C}^{\alpha} \mathrm{CO}}^{2}\right\rangle_{o}=0.955 \pm 0.001$ when flexible tails and loops are excluded from the evaluation. These values are consistent with the average model-free order parameter $S_{L S}^{2}$ for $\mathrm{NH}$ obtained from laboratory frame relaxation measurements (Hall and Fushman 2003; Tjandra et al. 1995). The fluctuations of $\mathrm{C}^{\alpha} \mathrm{CO}$ bonds are being investigated by cross correlated relaxation measurements (Chang and Tjandra 2005; Ferrage et al. 2006; Wang et al. 2003). 
It follows that when $\vartheta^{2}$ is determined using only the $\mathrm{NH}$ couplings the result should be slightly smaller than when only the $\mathrm{C}^{\alpha} \mathrm{CO}$ couplings are used, provided that no other motions play a role. Conversely, any additional motion will partially mask the intrinsic bond fluctuations and perturb the difference $\Delta=\left\langle\vartheta_{\mathrm{C}^{\alpha} \mathrm{CO}}^{2}\right\rangle-\left\langle\vartheta_{\mathrm{NH}}^{2}\right\rangle$. Asymmetric motions, i.e. motions that affect the two bonds unequally, will either increase or decrease this difference, or even keep it invariant, whereas symmetric motions will only decrease it. Any change in $\Delta$ that is due to the intrinsic secondary structure fluctuations, i.e. $S_{\alpha \beta}^{2}$, will be independent of the alignment medium. Any mediumdependent variation in $\Delta$ cannot arise from mediumindependent intrinsic molecular motions and must be attributed either to DMA or to some unknown, novel medium-induced motion.

In order to remove contributions from $S_{\tau}^{2}$ and such superfluous factors as LC-medium density and sample temperature that are present equally in $\vartheta_{\mathrm{NH}}^{2}$ and $\vartheta_{\mathrm{C}^{\alpha} \mathrm{CO}}^{2}$, we normalize $\Delta$ by $\vartheta_{\mathrm{C}^{\alpha} \mathrm{CO}}^{2}$ :

$\delta=\frac{\left\langle\vartheta_{\mathrm{C}^{\alpha} \mathrm{CO}}^{2}\right\rangle-\left\langle\vartheta_{\mathrm{NH}}^{2}\right\rangle}{\left\langle\vartheta_{\mathrm{C}^{\alpha} \mathrm{CO}}^{2}\right\rangle}=1-\frac{\left\langle\vartheta_{\mathrm{NH}}^{2}\right\rangle}{\left\langle\vartheta_{\mathrm{C}^{\alpha} \mathrm{CO}}^{2}\right\rangle}$

Furthermore we should note that the sample conditions of the data sets used in this study to illustrate DMA are so similar (cf. Clore and Schwieters 2004a, b; Cornilescu et al. 1998; Tossavainen et al. 2003; Ulmer et al. 2003) that we cannot see how they could have any significant effect on the results to be presented. Alignment tensors are determined from RDC data e.g. by using SVD (Losonczi et al. 1999). The $\delta$ parameter and its error are then obtained using Eq. 2.

\section{Amount of intrinsic bond fluctuations}

Based on the MD simulation derived $\left\langle\vartheta_{\mathrm{NH}}^{2}\right\rangle_{o}$ and $\left\langle\vartheta_{\mathrm{C}^{\alpha} \mathrm{CO}}^{2}\right\rangle_{o}$ values, the normalized difference between the average $\mathrm{NH}$ and $\mathrm{C}^{\alpha} \mathrm{CO}$ bond fluctuations is without any additional motion $\delta_{o}=0.081 \pm 0.002$. If the measured $\left\langle\vartheta_{\mathrm{C}^{\alpha} \mathrm{CO}}^{2}\right\rangle_{o}$ differs substantially from the simulated value of 0.955 , this value should be adjusted accordingly. It should also be noted that if the internal bond fluctuations were highly molecule dependent, it might be more sensible to consider $\delta_{\mathrm{o}}$ separately for each molecule rather than the general $\delta_{\mathrm{o}}$ shown here. Nevertheless, a $\delta_{\mathrm{o}}$ parameter derived from a system outside the influence of any alignment medium serves to establish a numeric value that includes all intrinsic motions and nothing else. Any deviation from this baseline is then a sign of additional motions.
Local alignment frames

It may appear strange to use the general degree of order, a molecular quantity, as a motional probe because local order parameters are more informative. A locus-specific measure of RDC-probed dynamics is obtained by combining, via transformations, a non-redundant data set of at least five alignments (Tolman et al. 2001). The obtained local GDOs provide a clear measure of residue-specific dynamics, but unfortunately the procedure would also mix up the eventual fluctuations in alignments-the very effect we aim to address. To differentiate between internal and alignment fluctuations it only matters to observe whether $\delta$ varies from one medium to another. To this end $\vartheta^{2}$ is a robust measure that samples various bond directions. By taking care to account for alignment frame fluctuations and population differences between the conformations (Louhivuori et al. 2006), one could arrive at more precise local order parameters.

\section{Methods}

The conformational ensembles of GB1 (850 members), GB3 (150 members) and ubiquitin (2,400 members) were generated by molecular dynamics using the CHARMM22 parameter set (MacKerell et al. 1998) with a modified backbone potential (MacKerell et al. 2004) and generalized Born model (Im et al. 2003). The proteins were first heated to $300 \mathrm{~K}$ during $200 \mathrm{ps}$ and then equilibrated for $2 \mathrm{~ns}$ at this temperature before sampling for 48, 34 and $3 \mathrm{~ns}$ for ubiquitin, GB1 and GB3, respectively. Subsequently the alignment of each conformation was simulated using the PALES program (Zweckstetter 2006; Zweckstetter and Bax 2000; Zweckstetter et al. 2004) in order to achieve the alignment dispersion present in a real sample. The alignments were predicted in the presence of a neutral planar obstacle as well as using positively and negatively charged bacteriophages carrying a surface charge density $0.047 \mathrm{e} / \mathrm{nm}^{2}$ and $10 \mathrm{mM}$ ionic strength of monovalent salt in $\mathrm{pH} 6.5$ with a LC concentration of $1 \mathrm{mg} / \mathrm{ml}$.

The refined structures 1P7F (Clore and Schwieters 2004a; Ulmer et al. 2003), 1D3Z (Clore and Schwieters 2004b; Cornilescu et al. 1998) and 1NYA (Tossavainen et al. 2003) of the B3 domain of protein G, ubiquitin and calerythrin were used to analyse experimental RDC data available in various LC media (Table 1). The temperature dependence of the experimental parameters were reported (Cornilescu et al. 1998; Ulmer et al. 2003) to be minimal in the temperature range used in the experiments. 
Table $1 \vartheta^{2}$ and $\delta$ of GB3, ubiquitin and calerythrin in various alignment media. $\vartheta^{2}$ are shown in units of $10^{-6}$

\begin{tabular}{|c|c|c|c|c|c|}
\hline Protein & Medium & $\left\langle\vartheta_{\mathrm{NH}}^{2}\right\rangle$ & $\left\langle\vartheta_{\mathrm{C}^{\alpha} \mathrm{CO}}^{2}\right\rangle$ & $\delta \pm \Delta \delta$ & $\mathrm{T}(\mathrm{K})$ \\
\hline \multirow[t]{5}{*}{ GB3 $(1 \mathrm{P} 7 \mathrm{~F})^{\mathrm{a}}$} & CTAB & 2.073 & 2.349 & $0.117 \pm 0.006$ & 302 \\
\hline & PEG & 0.614 & 0.750 & $0.181 \pm 0.009$ & 304 \\
\hline & PAG+ & 1.079 & 1.242 & $0.131 \pm 0.007$ & 298 \\
\hline & PAG- & 1.113 & 1.320 & $0.158 \pm 0.006$ & 298 \\
\hline & Pf1 & 1.377 & 1.731 & $0.205 \pm 0.005$ & 302 \\
\hline \multirow[t]{2}{*}{ UBI (1D3Z) ${ }^{\mathrm{b}}$} & Bicelles & 0.695 & 0.788 & $0.118 \pm 0.005$ & 304 \\
\hline & CTAB & 2.269 & 2.685 & $0.155 \pm 0.003$ & 304 \\
\hline CAL $(1 \mathrm{NYA})^{\mathrm{c}}$ & Pf1 & 4.451 & 4.728 & $0.059 \pm 0.001$ & 318 \\
\hline
\end{tabular}

Experimental temperature is shown for an easy comparison with $\delta$ variation

${ }^{\mathrm{a}} \mathrm{pH} 6.5, \varepsilon_{\mathrm{NH}}=0.26 \mathrm{~Hz}, \varepsilon_{\mathrm{C}^{\alpha} \mathrm{CO}}=0.10 \mathrm{~Hz} ;$ Pf1 $11 \mathrm{mg} / \mathrm{ml}, 100 \mathrm{mM} \mathrm{NaCl}$

b $\varepsilon_{\mathrm{NH}}=0.15 \mathrm{~Hz}$

c $\mathrm{pH}$ 6.0; Pf1 $30 \mathrm{mg} / \mathrm{ml}$

An effective alignment tensor for each ensemble was calculated using SVD (Losonczi et al. 1999). Either the experimental or average PALES predicted RDCs were used. Besides the refined structures (Table 1) experimental RDCs were used also with the MD simulated-PALES aligned structures in what we call the hybrid model (Table 5). PALES predicted average RDCs were used in conjunction with the MD simulated-PALES aligned structures (Table 2).

\section{Results and discussion}

Analysis of experimental data

The wealth of high-quality residual dipolar coupling data of the B3 domain of protein G (GB3) and ubiquitin (UBI) and their refined structures (1P7F and 1D3Z, respectively) allow us to determine GDOs and to calculate $\delta$ values for several cases (Table 1). The data for calerythrin (1NYA), a significantly larger protein ( $\sim 20 \mathrm{kDa}$. less than $10 \mathrm{kDa})$, is also available (Tossavainen et al. 2003). The quality of the refined structures in terms of an RMSD measure, referred to as the quality factor (Clore and Garrett 1999), is in all these cases good. Accordingly, in the evaluation of $\delta$ we employ only the $\mathrm{NH}$ and $\mathrm{C}^{\alpha} \mathrm{CO}$ couplings that were used to refine the GB3, ubiquitin and calerythrin structures. The GDO values were calculated from the experimental dipolar coupling data using a SVD algorithm against the average structures of the refined ensembles.

The two most important observations are that $\delta>\delta_{o}$ and that $\delta$ varies from one medium to another. Therefore it seems that there are motions in addition to bond fluctuations and we attribute them, owing to their mediumdependency, at least partly to dynamics modulated alignment.

The results are subject to the following uncertainties. The errors in $\delta$ were obtained by a Monte-Carlo (MC) method from the experimental errors in RDCs. The largest ambiguity originates from the ratio between the $\mathrm{NH}$ and

Table $2 \Omega$ and $\delta$ parameters for simulated conformational ensembles of the B1 and B3 domain of protein G and human ubiquitin based on PALES-predicted average RDCs

\begin{tabular}{llcccr}
\hline Protein & Medium & \multicolumn{1}{c}{$\Omega_{\mathrm{NH}}\left(^{\circ}\right)$} & $\Omega_{\mathrm{C}^{\alpha} \mathrm{CO}}\left(^{\circ}\right)$ & $\delta_{\text {direct }}$ & $\delta_{\mathrm{SVD}}$ \\
\hline GB1 & Negative & $31.7 \pm 0.2$ & $31.1 \pm 0.2$ & $0.172 \pm 0.007$ & $0.109 \pm 0.008$ \\
& Neutral & $11.82 \pm 0.04$ & $7.38 \pm 0.02$ & $0.114 \pm 0.001$ & $0.116 \pm 0.008$ \\
& Positive & $40.36 \pm 0.05$ & $36.23 \pm 0.03$ & $0.385 \pm 0.004$ & $0.208 \pm 0.002$ \\
GB3 & Negative & $12.60 \pm 0.10$ & $9.54 \pm 0.06$ & $0.082 \pm 0.003$ & $0.097 \pm 0.009$ \\
& Neutral & $11.82 \pm 0.09$ & $7.75 \pm 0.05$ & $0.096 \pm 0.003$ & $0.100 \pm 0.009$ \\
& Positive & $23.0 \pm 0.4$ & $20.6 \pm 0.4$ & $0.119 \pm 0.010$ & $0.107 \pm 0.007$ \\
UBI & Negative & $13.21 \pm 0.04$ & $9.87 \pm 0.02$ & $0.090 \pm 0.001$ & $0.106 \pm 0.003$ \\
& Neutral & $16.95 \pm 0.09$ & $13.16 \pm 0.08$ & $0.080 \pm 0.002$ & $0.085 \pm 0.005$ \\
& Positive & $15.84 \pm 0.06$ & $12.86 \pm 0.05$ & $0.092 \pm 0.002$ & $0.104 \pm 0.007$ \\
\hline
\end{tabular}


$\mathrm{C}^{\alpha} \mathrm{CO}$ bond length. We used $r_{\mathrm{NH}}=1.02 \AA$ (Jeffrey 1992, p. 270) and $r_{\mathrm{C}^{\alpha} \mathrm{CO}}=1.53 \AA$ (Engh and Huber 1991; Lehmann et al. 1972). An eventual error in the bond length ratio is systematic and affects the absolute $\delta$ value, but it does not abolish the variation in $\delta$ from one medium to another. It should be noted that customarily when a structure is refined against RDCs $r_{\mathrm{NH}}$ is set to $1.04 \AA$ (Ottiger and Bax 1998) to obtain the average directions of the more mobile amides consistent with less mobile bonds, e.g. those of the $\mathrm{C}^{\alpha} \mathrm{CO}$ vectors. In other words, it is desirable to obtain the same magnitude of alignment tensor irrespective of the couplings employed. Further uncertainty stems from the discrete sampling of directions by the nonuniform and dissimilar distributions of $\mathrm{NH}$ and $\mathrm{C}^{\alpha} \mathrm{CO}$ bonds, but as will be shown later on it does not seem to matter that much. Furthermore, as mentioned above, no motional effects including the consequences of discrete non-uniform sampling can be seen in $\delta$ when the excess fluctuations are small compared to $S_{L S}^{2}$. Large alignment fluctuations on the other hand lead categorically to nearly uniform sampling of directions.

\section{Simple model of wobble}

A simple approximation of internal bond motions considers fluctuations about the mean direction only as axial, i.e. including just the zero-order spherical harmonics $Y_{00}$.

$\vartheta^{2}=\frac{4 \pi}{5} \sum_{m=-2}^{2}\left\langle Y_{0 m} Y_{0 m}^{*}\right\rangle \approx \frac{1}{4}\left(3\left\langle c^{2}\right\rangle_{\theta, \Omega}-1\right)^{2}$

Assuming that the internal bond $(\theta)$ and alignment $(\Omega)$ fluctuations are independent and on different timescales, they can be separated and treated independently (Brainard and Szabo 1981; Petersen and Chan 1977). If an isotropic sampling of bond directions is also assumed, then the combined effect of the fluctuations will be $S_{\theta \Omega}=S_{\theta} S_{\Omega}$. It would then be clear from Eq. 2 that $\delta$ values remain invariant even in the presence of alignment fluctuations.

However, it is evident from Table 1 that the $\delta$ parameter varies from one medium to another implying a coupling between the alignment fluctuations and $\delta$. It is therefore reasoned that $S_{\theta \Omega}$ cannot be factorised. This is not a contradiction since neither of the previously mentioned assumptions are entirely valid in this case. (i) The two fluctuations are not completely independent since there is a slight coupling between the alignment and the ps-ns bond motions (Louhivuori et al. 2006). (ii) Since alignment fluctuations are not a real motion, but rather a manifestation of the state space available in the system, alignment fluctuations can be said to affect all time-scales.
Therefore we propose the following simple ad hoc model to describe the combined effect of internal bond motions and alignment frame fluctuations.

$\vartheta^{2} \approx \frac{1}{4}\left[3 \cos ^{2}\left(\theta_{o}+\Omega\right)-1\right]^{2}$

Essentially this means that we are considering a model where a molecule's wobble about an average orientation amplifies the effect of internal bond motions. As a consequence the effective opening angle of internal bond motions is increased from the initial value of $\theta_{o}$ by $\Omega$.

In the absence of alignment fluctuations $(\Omega=0) \theta_{o}$ can be calculated using Eq. 5 from the GDO values determined for the MD simulated ensembles.

$\left\langle\vartheta^{2}\right\rangle_{o} \approx \frac{1}{4}\left[3 \cos ^{2}\left(\theta_{o}\right)-1\right]^{2}$

Accordingly, $\theta_{\mathrm{o}}$ for $\mathrm{NH}$ and $\mathrm{C}^{\alpha} \mathrm{CO}$ were calculated from the previously mentioned MD-derived $\left\langle\vartheta_{\mathrm{NH}}^{2}\right\rangle_{o}$ and $\left\langle\vartheta_{\mathrm{C}^{\alpha} \mathrm{CO}}^{2}\right\rangle_{o}$ and found to be $11.82^{\circ}$ and $9.99^{\circ}$, respectively. Then $\left\langle\vartheta_{\mathrm{NH}}^{2}\right\rangle$ and $\left\langle\vartheta_{\mathrm{C}^{\alpha} \mathrm{CO}}^{2}\right\rangle$ were calculated using Eq. 4 and plotted as functions of increasing $\Omega$ (Fig. 2). Finally, $\delta$ versus $\Omega$ was calculated from $\left\langle\vartheta_{\mathrm{NH}}^{2}\right\rangle$ and $\left\langle\vartheta_{\mathrm{C}^{\alpha} \mathrm{CO}}^{2}\right\rangle$ using Eq. 2 .

When the internal bond and alignment fluctuations combined reach the magic angle the alignment is lost all together. The normalized difference in the $\mathrm{NH}$ and $\mathrm{C}^{\alpha} \mathrm{CO}$ probed order, denoted by $\delta$, is available from measurements and shown in Fig. 2 for the B3 domain of protein

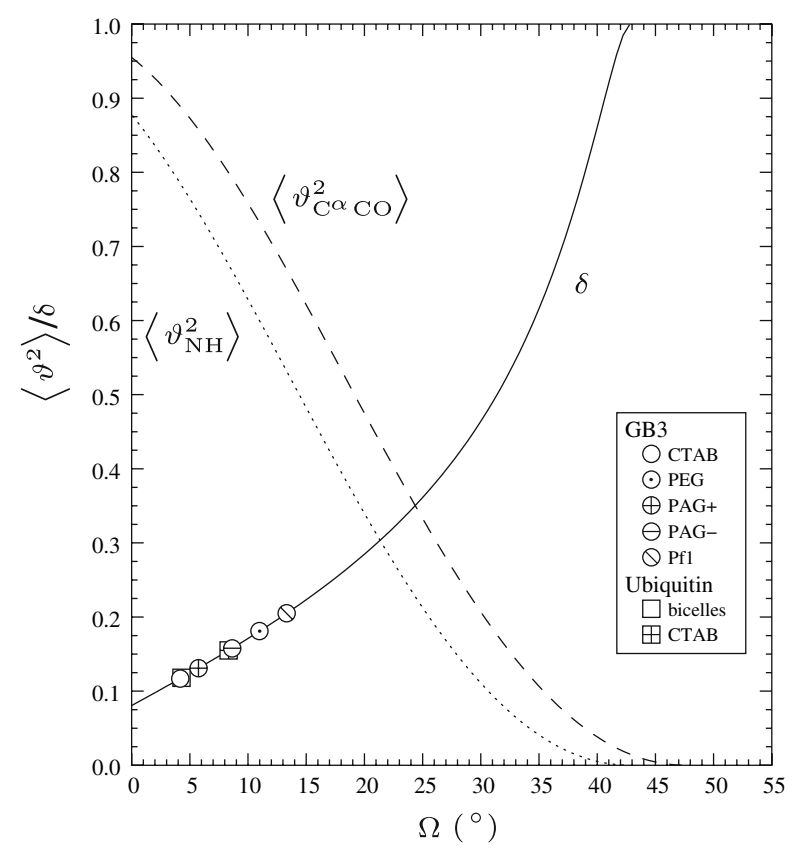

Fig. 2 Calculated effective order parameters $\left\langle\vartheta^{2}\right\rangle$ for the $\mathrm{NH}$ (dotted) and $\mathrm{C}^{\alpha} \mathrm{CO}$ (dashed) bonds and the $\delta$ parameter calculated from them as the molecule is subject to a fluctuating alignment of increasing angle $\Omega$ in addition to internal bond fluctuations 
$\mathrm{G}$ in CTAB doped bicelles, positively and negatively charged stretched polyacrylamide gels, polyethylene-glycol lamella and filamentous phages, and for ubiquitin in bicelles and CTAB doped bicelles. The $\delta$ value of calerythrin was smaller than the proposed general minimum of 0.081 and is therefore omitted. The symbols in the figure are larger than the errors obtained by MC analysis. It needs to be stressed that $\Omega$ should not be interpreted as a real measure of molecular motion since alignment fluctuations are only an ensemble property affecting RDC data.

When the experimental $\delta$ values are placed on the $(\delta, \Omega)$ curve we see that for both GB3 and ubiquitin in bicelle medium the effect of DMA is small enough to be comparable to that of bond fluctuations. Non-axial bond fluctuations will naturally complicate the situation and might invalidate such a comparison. The precision in $\delta$ is modest owing to the functional form of the $(\delta, \Omega)$-curve. In other media, particularly in those carrying surface charges on the nematogen, the alignments fluctuate noticeably. For example in filamentous phages the alignment of GB3 fluctuates even more than $13^{\circ}$. Thus $\vartheta^{2}$ may fall even below 0.7 . However, this does not mean that the molecule is somehow reorienting itself with such large-amplitude motions. It only tells that quite likely there are more than one major alignment modes separated from each other sufficiently to give the impression of large-scale motions if the alignment fluctuations are interpreted simply as real, molecular fluctuations around an average direction as was done in Eq. 4. Nonetheless, the medium-dependent variation in $\delta$ shows that DMA contributes to RDC-probed dynamics.

\section{Analysis of simulation data}

The sources of the medium-dependent variation in $\delta$ found from the experimental data can be many. Fortunately, they can be assessed via computer simulations. We consider the variation in the protein shape and charge distribution due to the ps-ns dynamics. These fluctuations give rise to a family of conformations whose members do not all align in exactly the same way. Simultaneous variation in the shape and net electric dipole moment may lead to a substantial alignment fluctuation. However, it is also conceivable that dynamics of the nematogen contributes to the protein alignment dispersion. Other microscopic heterogeneity in obstructing nematogens may also add to the variation of protein alignments but we are unequipped to examine these effects. Neither are the possible effects of hydration fully explored in this study.

The average $\mathrm{NH}$ and $\mathrm{C}^{\alpha} \mathrm{CO}$ RDCs of the simulated conformational ensembles were calculated and the GDO values were determined using SVD against the average structure of the ensemble. The average of the cosine matrix $c_{i} c_{j}$ of Eq. 1 was taken to present the best possible average structure. In this way we aimed to mimic the experimental procedure where GDOs were determined from the measured couplings against the refined structure. Alternatively, the knowledge of all $\mathrm{NH}$ and $\mathrm{C}^{\alpha} \mathrm{CO}$ vectors of the dispersed ensembles allowed us to calculate directly $\left\langle c^{2}\right\rangle_{\theta, \Omega}$ of Eq. 3 and hence also the order parameters and $\delta$ for the various cases. The two methods, direct and SVD, give similar $\delta$ values, with only GB1 in positive alignment medium showing a larger discrepancy (Table 2). Since both exhibit similar overall tendencies the methods can be assumed to be qualitatively consistent.

The alignment simulations in the neutral, positively and negatively charged media were not intended to match specifically any of the experimental conditions, but nevertheless the values are somewhat comparable. The simulated $\delta$ values are more uniform, but comparable in size (Table 2). Only the B1 domain of protein $\mathrm{G}$ seems to be significantly susceptible to DMA. The lack of dispersion in $\delta$ was unexpected in light of the clear dispersion found from experimental data (Table 1). Because of this and since the limited sampling of GB3 leaves a shadow of doubt on the exact values reported in Table 2, further work was done to understand the source for the lack of dispersion in $\delta$. First, non-uniform sampling of bond directions was addressed. Second, the possible increase in PALESpredicted alignment dispersion due to hydration was considered. Third, a hybrid model was used to circumvent any shortcomings in PALES-predicted RDCs.

Non-uniform bond direction sampling

The finite sampling of bond directions may influence the $\delta$ parameter. Therefore, we calculated the fraction of vectors oriented along the three principal directions as suggested by Fushman et al. (2000). The fractions $f_{\mathrm{i}}$ are a convenient measure of the uniformity of the bond direction sampling. In the case of uniform sampling $f_{\mathrm{z}}=f_{\mathrm{y}}=f_{\mathrm{x}}=1 / 3$, whereas a significant deviation from $1 / 3$ is a sign of nonuniform sampling. The Fushman-fractions calculated from the MD ensembles show gratifyingly that the sampling is rather uniform (Table 3).

Nevertheless, the possible effects of discrete, nonuniform sampling of bond directions was tested using the $\mathrm{MD}$ ensembles. The $\mathrm{C}^{\alpha} \mathrm{CO}$ bonds of every conformation were first rotated one residue at a time to point, on average, at the same direction as the $\mathrm{NH}$ bonds did, on average. A new set of RDCs was then calculated using the twisted structures and the original Saupe matrices. Singular value decomposition was then used to obtain an effective GDO value using the twisted structures and the new average RDCs. From the $\mathrm{NH}$ and $\mathrm{C}^{\alpha} \mathrm{CO}$ GDO values the $\delta$ parameter was calculated using Eq. 2. As can be seen from 
Table 3 The fraction of vectors oriented along the three principal axis calculated for the MD simulated ensembles of GB1, GB3 and ubiquitin

\begin{tabular}{lllll}
\hline Protein & Bond & $f_{\mathrm{z}}$ & $f_{\mathrm{y}}$ & $f_{\mathrm{x}}$ \\
\hline GB1 & $\mathrm{NH}$ & 0.54 & 0.26 & 0.20 \\
& $\mathrm{C}^{\alpha} \mathrm{CO}$ & 0.45 & 0.29 & 0.26 \\
$\mathrm{~GB} 3$ & $\mathrm{NH}$ & 0.55 & 0.26 & 0.19 \\
& $\mathrm{C}^{\alpha} \mathrm{CO}$ & 0.45 & 0.29 & 0.26 \\
$\mathrm{UBI}$ & $\mathrm{NH}$ & 0.49 & 0.31 & 0.21 \\
& $\mathrm{C}^{\alpha} \mathrm{CO}$ & 0.37 & 0.34 & 0.29 \\
\hline
\end{tabular}

Table 4, $\delta$ parameters changed, but mostly within the margins of error. Both GB1 in positively charged medium and ubiquitin in neutral medium experience a sharp drop in $\delta$. It seems that this is due to the fact that the $\mathrm{C}^{\alpha} \mathrm{CO}$ bonds rotated pre-dominantly closer to the magic angle diminishing $\mathrm{C}^{\alpha} \mathrm{CO}$ couplings sufficiently to render them much more labile in the face of the applied MC error. However, non-uniform bond direction sampling does not seem to influence $\delta$ significantly in most cases.

\section{Hydration shell}

Since the MD simulated conformational ensembles seemed to be less susceptible to DMA than NMR-based ensembles, it was plausible that PALES failed to impose a realistic dispersion of alignments. We hypothesised that the addition of a hydration shell to the molecules would make them more globular and therefore more susceptible to alignment dispersion due to steric interactions. To test whether this hypothesis could account for the observed lack of dispersion, a hydration layer needed to be added to the molecules. This was done with a rough model of the hydration shell in the form of increased atomic radii in PALES. Atomic radii were increased by $1,2,3$, and $4 \AA$, but no increase in DMA was observed neither for GB1 nor for ubiquitin. The lack of a hydration shell does not therefore seem to be the cause

Table 4 Alignment fluctuations in the MD ensemble before $\left(\delta_{\mathrm{SVD}}\right)$ and after $\left(\delta_{\text {SVD }}^{\prime}\right) \mathrm{C}^{\alpha} \mathrm{CO}$ bonds were superimposed on $\mathrm{NH}$ bonds

\begin{tabular}{llll}
\hline Protein & Medium & $\delta_{\text {SVD }}$ & $\delta_{\text {SVD }}^{\prime}$ \\
\hline GB1 & Negative & 0.109 & $0.113 \pm 0.008$ \\
& Neutral & 0.116 & $0.116 \pm 0.007$ \\
& Positive & 0.208 & $0.130 \pm 0.008$ \\
GB3 & Negative & 0.097 & $0.116 \pm 0.006$ \\
& Neutral & 0.100 & $0.106 \pm 0.006$ \\
& Positive & 0.107 & $0.113 \pm 0.006$ \\
UBI & Negative & 0.106 & $0.109 \pm 0.003$ \\
& Neutral & 0.085 & $0.018 \pm 0.008$ \\
& Positive & 0.104 & $0.092 \pm 0.007$ \\
\hline
\end{tabular}

for the difference in DMA between simulations and experiments.

\section{Hybrid model}

Another possibility was that PALES dispersed the alignments sufficiently, but that the predicted RDCs had some issues that were reflected in the effective alignment and ultimately in $\delta$. To overcome this, we calculated the effective alignments of the MD ensembles using experimental RDCs instead of PALES-predicted ones. This was done for GB3 in Pf1 and CTAB-doped bicelle media and for ubiquitin in bicelle media with and without CTAB. As can be seen from Table 5 and Fig. 3 this seems to be the

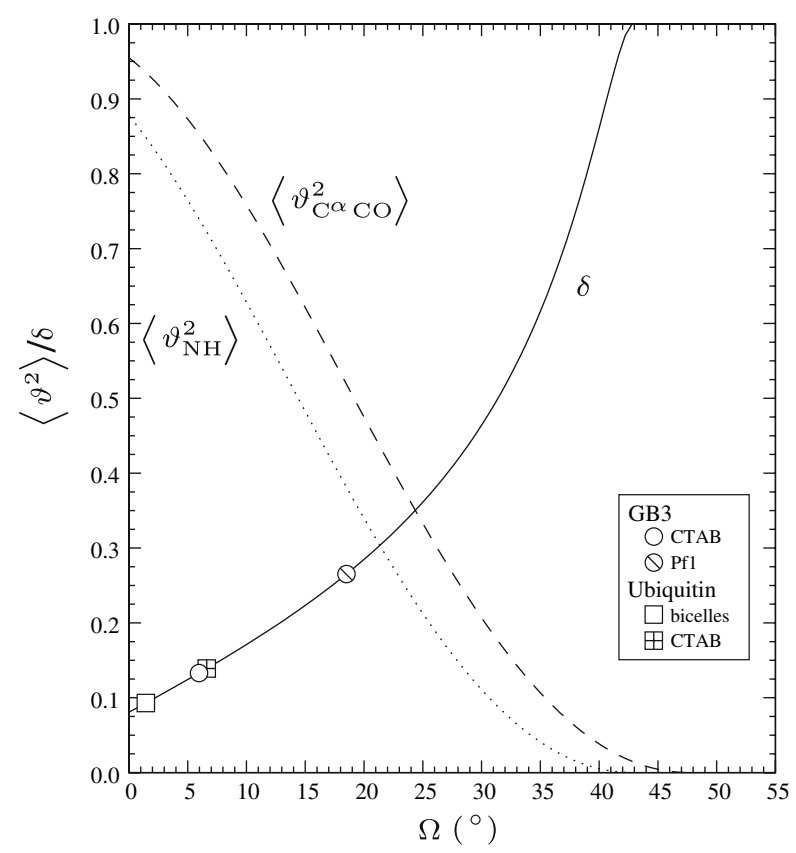

Fig. 3 Hybrid model. Calculated effective order parameters $\left\langle\vartheta^{2}\right\rangle$ for the $\mathrm{NH}$ (dotted) and $\mathrm{C}^{\alpha} \mathrm{CO}$ (dashed) bonds and the $\delta$ parameter calculated from them as the MD simulated conformations are subject to a fluctuating alignment of increasing angle $\Omega$ in addition to internal bond fluctuations

Table 5 Hybrid model

\begin{tabular}{llll}
\hline Protein & Medium & $\delta$ & $\delta_{\text {hybrid }}$ \\
\hline GB3 & CTAB & 0.117 & $0.133 \pm 0.005$ \\
& Pf1 & 0.205 & $0.265 \pm 0.004$ \\
UBI & Bicelles & 0.118 & $0.093 \pm 0.008$ \\
& CTAB & 0.155 & $0.139 \pm 0.006$
\end{tabular}

Alignment fluctuations in simulated conformational ensembles of the B3 domain of protein $\mathrm{G}$ and human ubiquitin based on experimental RDCs $\left(\delta_{\text {hybrid }}\right)$ compared to those in the refined, experimental structures $(\delta)$ 
major cause for the lack of $\delta$-dispersion. What this suggests is that when experimental RDCs are available DMA can be estimated consistently from a MD-simulated conformational ensemble even without structures refined against experimental RDCs.

\section{Competition of interactions}

In the neutral medium only the molecular shape fluctuations contribute and DMA is comparable to the internal bond vector fluctuations. Neither in the simulation nor in the experimental data is there a lot of difference between the smaller molecules GB3 and ubiquitin. Calerythrin resembles more an oblate ellipsoid (Fig. 4) and is expected to fluctuate less than the smaller, globular proteins in the absence of electrostatic interactions.

It is more intricate to rationalize the reduced order parameters in a charged media as both molecular shape and charge distribution fluctuations are present. It may not be possible to simplify the dynamics of a complex set of interactions to provide lucid understanding to the causes of DMA. To begin with, GB1, GB3, ubiquitin and calerythrin carry under experimental conditions a net charge of -1.22 ,
$-3.09,+1.39$ and -8.45 , respectively. In the repulsive electrostatic potential the alignment is, to a crude approximation, governed by the direction and size of the net electric dipole moment in relation to the molecular shape tensor.

A competition between the steric and electrostatic alignment arises when the dipole moment is mostly along the long axis of the molecule and comparatively small. GB1 is a good example of this (Fig. 4). In GB1 dipole moments are pointing roughly to the middle of the long and short axis in two slightly separated groups. The situation is similar in GB3, but with the dipole moments concentrated in a single, tight group. Furthermore, the dipole moments in GB3 are larger than in GB1 and dominate the alignment. In the globular ubiquitin the dipole moments are directed along the shortest of the molecular axes. Thus both the shape and electrostatics act in synergy to narrow the cone of alignments. In spite of this the globular shape of ubiquitin ensures that modest alignment fluctuations remain in charged media. In the refined structures of calerythrin the dipole moments are spread out slightly more than in the MD simulated ensemble of GB1 and directed similarly roughly to the middle of the long and short axis. Alignment fluctuations in calerythrin are small (Table 1),
Fig. 4 Causes of molecular alignment fluctuations for (a) GB1, (b) GB3, (c) ubiquitin and (d) calerythrin illustrated for example conformations. The variation in the molecular shape and charge distribution are simplified by showing the molecular principle axes (grey) and net electric dipole moments (orange)
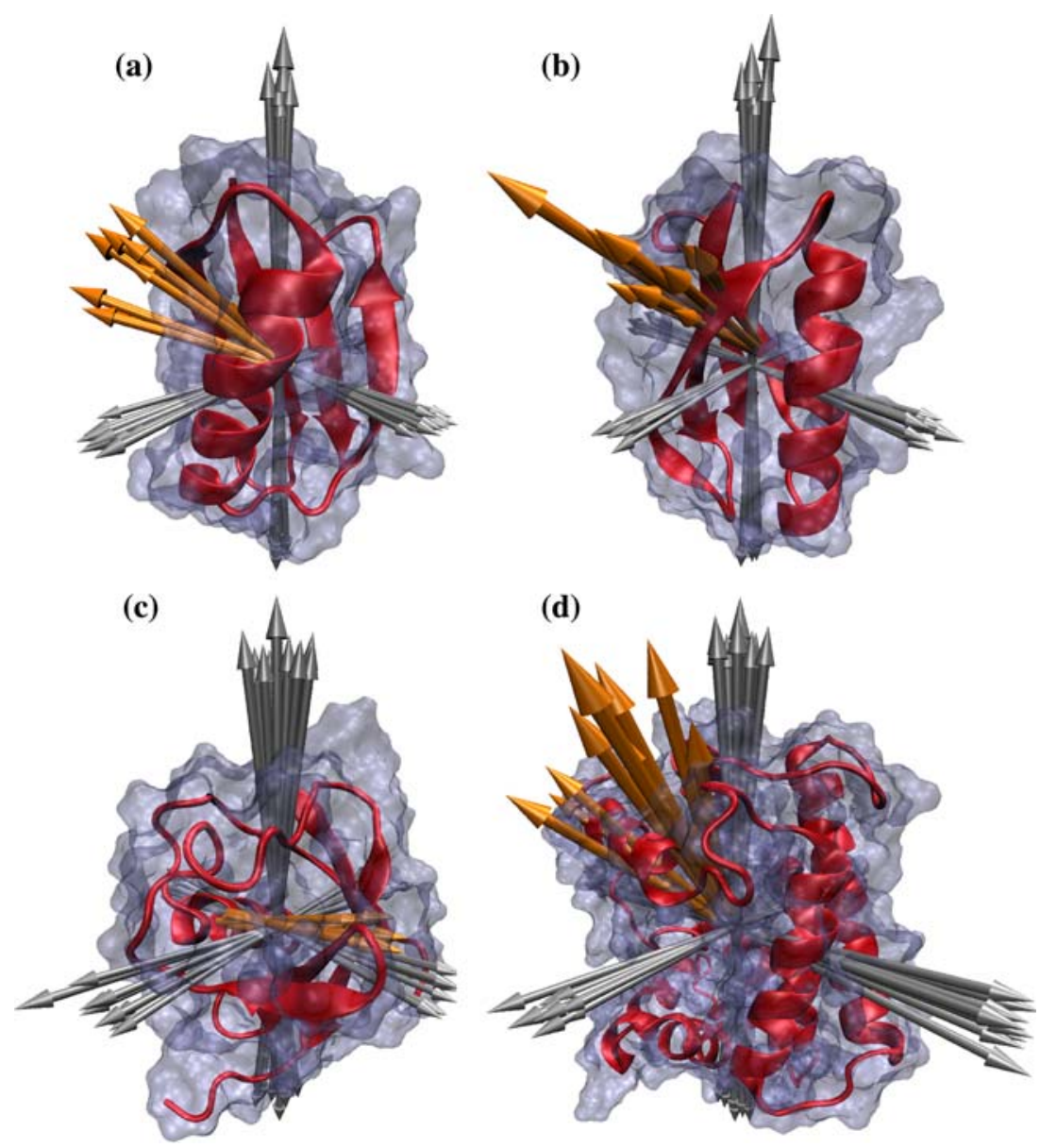
even though the combination of an oblate ellipsoidal shape with dipole moments directed away from the short axis suggest a competition between the steric and electrostatic alignments. Calerythrin contains a flexible hinge-region that has a slightly ambiguous structure in 1NYA (Tossavainen et al. 2003). It is thus possible that the lack of restraints in the structural refinement has led to somewhat more compact structures with slightly distorted net dipole moments. Analysis of an attractive potential is more involved and not easily reduced to the analysis of the net electric dipole moment relative to the shape tensor. The variation in the position of the net charge influences the alignment that can fluctuate substantially as exemplified by the aforementioned $\delta$ values for GB3.

In general a small globular protein that carries many charges and yet only a small net electric dipole moment is the most vulnerable to molecular alignment fluctuations. The shape as well as the charge distribution is perturbed by dynamics of long side-chains at the molecular surface. For large proteins the effects are expected to have less impact on the overall molecular alignment.

\section{Conclusions}

Internal motions are, at least to a good approximation, medium-independent. Therefore one would expect that e.g. relative differences between $\mathrm{NH}$ and $\mathrm{C}^{\alpha} \mathrm{CO}$ bond fluctuations, denoted by the $\delta$ parameter, would remain the same from one medium to another. However, we find that this is not the case for protein $\mathrm{G}$ and ubiquitin. The changes in $\delta$ from one medium to another imply the presence of an additional medium-dependent ensemble property involved in the alignment phenomenon.

The simulations suggest that the effective alignment of a molecule fluctuates as a response to the variation in molecular shape and charge distribution caused by protein dynamics. This alignment modulation is at its highest when steric and electrostatic interactions compete for the dominance in a small globular protein. Also flexible loops and termini may cause a fluctuating alignment. The alignment fluctuation as a phenomenon is known and e.g. used to point out domain-domain motions in modular proteins (Bewley and Clore 2000; Fischer et al. 1999) and flexibility of polypeptides (Chou et al. 2001; Goto et al. 2001).

Here we were able to consider only alignment effects due to protein dynamics that occur in the ps-ns range owing to the limitations of molecular dynamics simulations. Other states accessible via slower motions are of course also expected to contribute to the variation in molecular alignment depending on their population, shapes and charge distributions. We also expect that dynamics of nematogens and other variation in the media contribute as well to the bond vector dispersion underlying the observed RDC signals.

It is perhaps perplexing that molecular alignment fluctuations do not show up as clear inconsistencies in refinement of protein structures in particular when a number of RDCs obtained from measurements in various media are used simultaneously. It should be emphasized that the dynamics modulated alignment is a process that is nearly uncorrelated from the intrinsic vector fluctuations. Consequently it will, to an excellent approximation, only scale RDCs but leave their directional information content, used in the structure determination, intact. Thus the averaging due to DMA appears much as the averaging due to the molecular tumbling. Even a substantial wobble in the alignment can be compensated by increasing the strength of LC-medium to acquire large enough couplings for quantitative analyses. It should be stressed that the presence of DMA does not, for example, invalidate the recent finding (Bernadó et al. 2005) of long-range interactions in $\alpha$-Synuclein nor the previously mentioned supra- $\tau_{c}$ backbone motions, but gives a rationale for the low $S^{2}$ values. Perhaps indicative of fluctuating alignments are the challenges in predicting precisely RDC-data on the basis of a single structure.

In studies of dynamics where the dispersion of a vector direction rather than its average direction is of interest we expect that the alignment fluctuations are worth to consider as they will inevitably incorporate into the RDC-based order parameter. In this study the evidence of molecular alignment fluctuation was established using the average molecular parameter, general degree of order. Consequently the site-specific variation in the order and its correlation to the secondary structure that have been reported in the RDC-probed dynamics studies was not addressed. It is conceivable that an alignment fluctuation could result in some site-specific variation in $\vartheta^{2}$ depending on the direction of the bonds, but unlikely in any correlated variations.

Acknowledgments We thank Kresten Lindorff-Larsen for providing the molecular dynamics simulated conformational ensembles. The study has been supported by the Academy of Finland and the National Technology Agency of Finland.

\section{References}

Bax A, Grishaev A (2005) Weak alignment NMR: a hawk-eyed view of biomolecular structure. Curr Opin Struct Biol 15:563-570

Bax A, Kontaxis G, Tjandra N (2001) Dipolar couplings in macromolecular structure determination. Methods Enzymol 339:127-174

Bernadó P, Blackledge M (2004) Local dynamic amplitudes on the protein backbone from dipolar couplings: toward the elucidation of slower motions in biomolecules. J Am Chem Soc 126:7760-7761 
Bernadó P, Bertoncini CW, Griesinger C, Zweckstetter M, Blackledge $M$ (2005) Defining long-range order and local disorder in native $\alpha$-Synuclein using residual dipolar couplings. J Am Chem Soc 127:17968-17969

Bewley CA, Clore GM (2000) Determination of the relative orientation of the two halves of the domain-swapped dimer of cyanovirin- $\mathrm{N}$ in solution using dipolar couplings and rigid body minimization. J Am Chem Soc 122:6009-6016

Blackledge M (2005) Recent progress in the study of biomolecular structure and dynamics in solution from residual dipolar couplings. Prog Nucl Magn Reson Spectrosc 46:23-61

Bouvignies G, Bernadó P, Meier S, Cho K, Grzesiek S, Brüschweiler R, Blackledge M (2005) Identification of slow correlated motions in proteins using residual dipolar and hydrogen-bond scalar couplings. Proc Natl Acad Sci USA 102:13885-13890

Brainard JR, Szabo A (1981) Theory for nuclear magnetic relaxation of probes in anisotropic systems: application to cholesterol in phospholipid vesicles. Biochemistry 20:4618-4628

Chang SL, Tjandra N (2005) Temperature dependence of protein backbone motion from carbonyl C-13 and amide N-15 NMR relaxation. J Magn Reson 174:43-53

Chou JJ, Li S, Klee CB, Bax A (2001) Solution structure of Ca(2+)calmodulin reveals flexible hand-like properties of its domains. Nat Struct Biol 8:990-997

Clore GM, Garrett DS (1999) R-factor, free R, and complete crossvalidation for dipolar coupling refinement of NMR structures. J Am Chem Soc 121:9008-9012

Clore GM, Schwieters CD (2004a) Amplitudes of protein backbone dynamics and correlated motions in a small alpha/beta protein: correspondence of dipolar coupling and heteronuclear relaxation measurements. Biochemistry 43:10678-10691

Clore GM, Schwieters CD (2004b) How much backbone motion in ubiquitin is required to account for dipolar coupling data measured in multiple alignment media as assessed by independent cross-validation? J Am Chem Soc 126:2923-2938

Cornilescu G, Marquardt JL, Ottiger M, Bax A (1998) Validation of protein structure from anisotropic carbonyl chemical shifts in a dilute liquid crystalline phase. J Am Chem Soc 120:6836-6837

Engh RA, Huber R (1991) Accurate bond and angle parameters for X-ray protein structure refinement. Acta Crystallogr A47:392-400

Ferrage F, Pelupessy P, Cowburn D, Bodenhausen G (2006) Protein backbone dynamics through $13 \mathrm{C}^{\prime}-13 \mathrm{C}$ crossrelaxation in NMR spectroscopy. J Am Chem Soc 128:11072-11078

Fischer MWF, Losonczi JA, Weaver JL, Prestegard JH (1999) Domain orientation and dynamics in multidomain proteins from residual dipolar couplings. Biochemistry 38:9013-9022

Fushman D, Ghose R, Cowburn D (2000) The effect of finite sampling on the determination of orientational properties: a theoretical treatment with application to interatomic vectors in proteins. J Am Chem Soc 122:10640-10649

Goto NK, Skrynnikov NR, Dahlquist FW, Kay LE (2001) What is the average conformation of bacteriophage T4 lysozyme in solution? A domain orientation study using dipolar couplings measured by solution NMR. J Mol Biol 308:745-764

Hall JB, Fushman D (2003) Characterization of the overall and local dynamics of a protein with intermediate rotational anisotropy: differentiating between conformational exchange and anisotropic diffusion in the B3 domain of protein G. J Biomol NMR 27: 261-275

Im W, Lee MS, Brooks I, Charles L (2003) Generalized Born model with a simple smoothing function. J Comput Chem 24:1691-1702

Jeffrey GA (1992) Accurate crystal structure analysis by neutron diffraction. In: Domenicano A, Hargittai I (eds) Accurate molecular structures-their determination and importance. Oxford University Press, Oxford, pp 270-298
Kay LE, Torchia DA, Bax A (1989) Backbone dynamics of proteins as studied by ${ }^{15} \mathrm{~N}$ inverse detected heteronuclear NMR spectroscopy: application to Staphylococcal nuclease. Biochemistry 28:8972-8979

Lakomek NA, Farés C, Becker S, Carlomagno T, Meiler J, Griesinger $\mathrm{C}$ (2005) Side-chain orientation and hydrogen-bonding imprint supra- $\tau_{c}$ motion on the protein backbone of ubiquitin. Angew Chem Int Ed 44:7776-7778

Lakomek NA, Carlomagno T, Becker S, Griesinger C, Meiler J (2006) A thorough dynamic interpretation of residual dipolar couplings in ubiquitin. J Biomol NMR 34:101-115

Lehmann MS, Koetzle TF, Hamilton WC (1972) Precision neutron diffraction structure determination of protein and nucleic acid components. I. The crystal and molecular structure of the amino acid L-Alanine. J Am Chem Soc 94:2657-2660

Lipari G, Szabo A (1982a) Model-free approach to the interpretation of nuclear magnetic resonance relaxation in macromolecules. 1 . Theory and range of validity. J Am Chem Soc 104:4546-4559

Lipari G, Szabo A (1982b) Model-free approach to the interpretation of nuclear magnetic resonance relaxation in macromolecules. 2. Analysis of experimental results. J Am Chem Soc 104: $4559-4570$

Losonczi JA, Andrec M, Fischer MWF, Prestegard JH (1999) Order matrix analysis of residual dipolar couplings using singular value decomposition. J Magn Reson 138:334-342

Louhivuori M, Otten R, Lindorff-Larsen K, Annila A (2006) Conformational fluctuations affect protein alignment in dilute liquid crystal media. J Am Chem Soc 128:4371-4376

MacKerell AD, Bashford D, Bellott M, Dunbrack RL, Evanseck JD, Field MJ, Fischer S, Gao J, Guo H, Ha S, Joseph-McCarthy D, Kuchnir L, Kuczera K, Lau FTK, Mattos C, Michnick S, Ngo T, Nguyen DT, Prodhom B, Reiher WE, Roux B, Schlenkrich M, Smith JC, Stote R, Straub J, Watanabe M, Wiorkiewicz-Kuczera J, Yin D, Karplus M (1998) All-atom empirical potential for molecular modeling and dynamics studies of proteins. J Phys Chem B 102:3586-3616

MacKerell AD, Feig M, Brooks CL (2004) Improved treatment of the protein backbone in empirical force fields. J Am Chem Soc 126:698-699

Meiler J, Prompers JJ, Peti W, Griesinger C (2001) Model-free approach to the dynamic interpretation of residual dipolar couplings in globular proteins. J Am Chem Soc 123:6098-6107

Meiler J, Peti W, Griesinger C (2003) Dipolar couplings in multiple alignments suggest $\alpha$ helical motion in ubiquitin. J Am Chem Soc 125:8072-8073

Millet O, Muhandiram DR, Skrynnikov NR, Kay LE (2002) Deuterium spin probes of side-chain dynamics in proteins. 1. Measurement of five relaxation rates per deuteron in 13C-labeled and fractionally $2 \mathrm{H}$-enriched proteins in solution. $\mathrm{J}$ Am Chem Soc 124:6439-6448

Ottiger M, Bax A (1998) Determination of relative $\mathrm{N}-\mathrm{H}^{N}, \mathrm{~N}-\mathrm{C}^{\prime}$, $\mathrm{C}^{\alpha}-\mathrm{C}^{\prime}$, and $\mathrm{C}^{\alpha}-\mathrm{H}^{\alpha}$ effective bond lengths in a protein by NMR in a dilute liquid crystalline phase. J Am Chem Soc 120: 12334-12341

Palmer AG (2004) NMR characterization of the dynamics of biomacromolecules. Chem Rev 104:3623-3640

Petersen NO, Chan SI (1977) More on the motional state of lipid bilayer membranes: interpretation of order parameters obtained from nuclear magnetic resonance experiments. Biochemistry $16: 2657-2667$

Peti W, Meiler J, Brüschweiler R, Griesinger C (2002) Model-free analysis of protein backbone motion from residual dipolar couplings. J Am Chem Soc 124:5822-5833

Prestegard JH, Bougault CM, Kishore AI (2004) Residual dipolar couplings in structure determination of biomolecules. Chem Rev 104:3519-3540 
Skrynnikov NR, Millet O, Kay LE (2002) Deuterium spin probes of side-chain dynamics in proteins. 2. Spectral density mapping and identification of nanosecond time-scale side-chain motions. J Am Chem Soc 124:6449-6460

Tjandra N, Bax A (1997) Direct measurement of distances and angles in biomolecules by NMR in a dilute liquid crystalline medium. Science 278:1111-1114

Tjandra N, Feller SE, Pastor RW, Bax A (1995) Rotational diffusion anisotropy of human ubiquitin from 15N NMR relaxation. J Am Chem Soc 117:12562-12566

Tolman JR (2002) A novel approach to the retrieval of structural and dynamic information from residual dipolar couplings using several oriented media in biomolecular NMR spectroscopy. J Am Chem Soc 124: 12020-12030

Tolman JR, al Hashimi HM, Kay LE, Prestegard JH (2001) Structural and dynamic analysis of residual dipolar coupling data for proteins. J Am Chem Soc 123:1416-1424

Tossavainen H, Permi P, Annila A, Kilpelainen I, Drakenberg T (2003) NMR solution structure of calerythrin, an EF-hand calcium-binding protein from Saccharopolyspora erythraea. Eur J Biochem 270:2505-2512

Ulmer TS, Ramirez BE, Delaglio F, Bax A (2003) Evaluation of backbone proton positions and dynamics in a small protein by liquid crystal NMR spectroscopy. J Am Chem Soc 125: 9179-9191

Wang T, Cai S, Zuiderweg ERP (2003) Temperature dependence of anisotropic protein backbone dynamics. J Am Chem Soc 125:8639-8643

Zweckstetter M (2006) Prediction of charge-induced molecular alignment: residual dipolar couplings at $\mathrm{pH} 3$ and alignment in surfactant liquid crystalline phases. Eur Biophys J 35:170-180

Zweckstetter M, Bax A (2000) Prediction of sterically induced alignment in a dilute liquid crystalline phase: aid to protein structure determination by NMR. J Am Chem Soc 122: 3791-3792

Zweckstetter M, Hummer G, Bax A (2004) Prediction of chargeinduced molecular alignment of biomolecules dissolved in dilute liquid-crystalline phases. Biophys J 86:3444-3460 